\title{
ARTICLE OPEN Hydrogen embrittlement of grain boundaries in nickel: an atomistic study
}

\author{
Shan Huang ${ }^{1}$, Dengke Chen ${ }^{1}$, Jun Song ${ }^{2}$, David L. McDowell ${ }^{1}$ and Ting Zhu ${ }^{1}$
}

The chemomechanical degradation of metals by hydrogen is widely observed, but not clearly understood at the atomic scale. Here we report an atomistic study of hydrogen embrittlement of grain boundaries in nickel. All the possible interstitial hydrogen sites at a given grain boundary are identified by a powerful geometrical approach of division of grain boundary via polyhedral packing units of atoms. Hydrogen segregation energies are calculated at these interstitial sites to feed into the Rice-Wang thermodynamic theory of interfacial embrittlement. The hydrogen embrittlement effects are quantitatively evaluated in terms of the reduction of work of separation for hydrogen-segregated grain boundaries. We study both the fast and slow separation limits corresponding to grain boundary fracture at fixed hydrogen concentration and fixed hydrogen chemical potential, respectively. We further analyze the influences of local electron densities on hydrogen adsorption energies, thereby gaining insights into the physical limits of hydrogen embrittlement of grain boundaries.

npj Computational Materials (2017)3:28; doi:10.1038/s41524-017-0031-1

\section{INTRODUCTION}

Challenges associated with a hydrogen economy are substantial, ranging from hydrogen generation and storage to transportation. ${ }^{1}$ Hydrogen in metallic containment systems, such as high-pressure vessels and pipelines can cause the degradation of their mechanical properties that can further result in a sudden and unexpected catastrophic fracture. ${ }^{2-5}$ A wide range of hydrogen embrittlement phenomena was attributed to the loss of cohesion of interfaces (between grains, inclusion and matrix, or phases) due to interstitially dissolved hydrogen. ${ }^{6}$ This concept and related models, ${ }^{7-9}$ however, have not been made sufficiently predictive due to a lack of fundamental understanding of the chemomechanical mechanisms of embrittlement, despite considerable research effort. $^{10-22}$ One challenge towards such an understanding is to establish the mechanistic connections between the mechanochemistry of hydrogen adsorption and the strength reduction of grain boundaries (GBs) with realistic atomic structure and under loading conditions pertinent to applications.

The structure of metallic materials can often be described by arrays of polyhedra consisting of closely packed clusters of atoms. These space-filling polyhedra result from the competition of maximizing the coordination number of metallic atoms, minimizing local free volumes, and avoiding repulsive atomic overlap. ${ }^{23}$ They can effectively serve as the basic structural units for analyzing interstices for absorption of $\mathrm{H}$ atoms. In this work, we adopt a powerful geometrical approach pioneered by Ashby et al. ${ }^{24-26}$ for analyzing the structure of GBs in terms of polyhedral packing units. We develop a corresponding computational route to effectively identify all the potential interstitial sites of $\mathrm{H}$ adsorption at GBs. By combining the atomistic calculations of $\mathrm{H}$ segregation energies at these interstitial sites and the thermodynamic theory of interfacial embrittlement, ${ }^{6-9}$ we evaluate how the structure of GBs influences the propensity of $\mathrm{H}$ chemisorption, as well as the consequent embrittlement of GBs. The results reveal the collective embrittlement effects due to surface adsorption and interfacial segregation of $\mathrm{H}$ atoms at multiple trapping sites. We further analyze the influences of local electron densities on the $\mathrm{H}$ adsorption energies at GBs and fractured surfaces, in order to understand the physical limits of $\mathrm{H}$ embrittlement of GBs.

We study the face-centered-cubic (fcc) Ni as a model system. An embedded atom method (EAM) potential is used to describe the $\mathrm{Ni}-\mathrm{H}$ system, ${ }^{27,}{ }^{28}$ and the total energy is given by

$E=\frac{1}{2} \sum_{i, j} \phi\left(r_{i j}\right)+\sum_{i} F_{i}\left(\rho_{i}\right)$,

where $\phi\left(r_{i j}\right)$ is the pair energy between atoms $i$ and $j$ which are separated by $r_{i j}$, $\rho_{i}$ is the electron density at atom $i$ caused by all of the other atoms in the system, $F_{i}\left(\rho_{i}\right)$ is the embedding energy of atom $i$ which depends on the host electron density $\rho_{i}$ at atom $i$. The detailed potential functions in Eq. (1) are described in refs. 27, 28. The fitting data of this EAM potential include the zero-point energy, which is important for $\mathrm{H}$ atoms. This potential has been benchmarked by the bulk, surface, and defect properties measured from experiments (Table 1). Note that the potential parameters were corrected, ${ }^{28}$ and the corresponding potential data and reference properties can be found at the website of the NIST Interatomic Potentials Repository Project. ${ }^{29}$

GBs studied in this work include the symmetric tilt boundaries of $\Sigma 5(310)[001]$ and $\Sigma 17(140)[001]$, as well as $\Sigma 11(113)[011]$ and $\Sigma 27(115)$ [011]. They are representative of different spatial arrangements of polyhedral packing units. In the following analysis of polyhedral packing units, we focus on $\Sigma 5(310)[001]$ and $\Sigma 17(140)[001] G B s$. The corresponding simulation cell consists of a bicrystal slab containing a single symmetric tilt $G B$ in the middle. The slab geometry is $3.3 \mathrm{~nm} \times 4.9 \mathrm{~nm} \times 1.4 \mathrm{~nm}(2112$ atoms) for $\Sigma 5(310)[001]$ and $2.9 \times 4.9 \times 1.4 \mathrm{~nm}$ (1840 atoms) for

\footnotetext{
${ }^{1}$ Woodruff School of Mechanical Engineering, Georgia Institute of Technology, Atlanta, GA 30332, USA and ${ }^{2}$ Department of Mining and Materials Engineering, McGill University, Montréal, QC H3A OC5, Canada

Correspondence: Ting Zhu (ting.zhu@me.gatech.edu)
}

Received: 18 February 2017 Revised: 10 June 2017 Accepted: 21 June 2017

Published online: 14 July 2017 
L17(140)[001], respectively. Periodic boundary conditions are imposed along both the out-of-plane and the in-plane horizontal directions in Fig. 1, while the slab is free to relax in the vertical direction to the GB. All the atomic structures studied are relaxed by conjugate gradient energy minimization.

It is known that a $\mathrm{H}$ atom embedded in the transition metal such as $\mathrm{Ni}$ behaves chemically like a $\mathrm{He}$ atom, such that the interstitially dissolved $\mathrm{H}$ in $\mathrm{Ni}$ generally exists in the form of $\mathrm{H}$ atoms instead of $\mathrm{H}_{2}$ molecules. ${ }^{30}$ According to Ashby et al., ${ }^{24}$ the interstitial trapping sites in the bulk and at GBs can be characterized by the polyhedral packing units of host atoms. Specifically, in a geometrical description of close packing of hard spheres of equal radii, the polyhedral hole refers to the void within the convex deltahedron, the vertices of which are the centers of neighboring atoms and the faces of which are equilateral triangles. Suppose a deltahedron is not further divisible in the sense that it is too small to accommodate another host atom. The number of distinguishable and indivisible deltahedra is limited to nine in three dimensions. The central hole of a deltahedron contains a large free volume and thus is an energetically favorable interstitial site for trapping the small $\mathrm{H}$ atom. ${ }^{10,31}$

\begin{tabular}{|lll|}
\hline Table 1. $H$ trapping sites and binding energy (eV per atom) \\
\hline & EAM potential & Experiment ${ }^{27}$ \\
\hline Molecular binding energy $E_{\mathrm{H}}$ & -2.37 & \\
Bulk: tetrahedron & -1.79 & -2.05 \\
octahedron & -2.20 & \\
(111) surface: top & -2.62 & -2.70 \\
three-fold & -2.71 & \\
\hline
\end{tabular}

We develop a computational route to uniquely divide a relaxed $\mathrm{GB}$ into deltahedral packing units by using a $\mathrm{H}$ atom as a probe. That is, a single $\mathrm{H}$ atom is embedded near a GB by assigning its initial position based on the nodal coordinates of a spatial mesh, with uniform nodal spacing of, for example, $0.1 \AA$. Then the $\mathrm{Ni}-\mathrm{H}$ system is relaxed using conjugate gradient energy minimization. To identify the type of the interstitial hole where the probing $\mathrm{H}$ atom sits, we determine the number of vertices of the deltahedron containing the probe $\mathrm{H}$ atom through the number of $\mathrm{Ni}$ atoms that coordinate with the central $\mathrm{H}$ atom with a cut-off radius of $2.65 \AA$, which is empirically set based on their equilibrium distance from the $\mathrm{Ni}-\mathrm{H}$ potential. The types of deltahedra identified have been verified by direct inspection of the atomic deltahedral structures enclosing the probing $\mathrm{H}$ atom via the visualization software of Atomeye. ${ }^{32}$

\section{RESULTS}

Polyhedral packing units

Figure 1 shows the relaxed atomic structures of $\Sigma 5(310)[001]$ and $\Sigma 17(140)[001]$ tilt GBs in pure $\mathrm{Ni}$, which have the same [001] tilt axis, but different tilt angles. The interstitial sites for $\mathrm{H}$ adsorption are identified using a single $\mathrm{H}$ atom as a probe as described above. It is seen that the bulk fcc lattice is simply composed of periodic tetrahedral and octahedral units. In contrast, the $\Sigma 5(310)$ [001] GB is composed of three types of polyhedral holes: bitetrahedron, pentagonal bi-pyramid, and capped trigonal prism; the $\Sigma 17(140)[001]$ GB involves an additional octahedral hole. In both GBs a capped trigonal prism always nests with a pentagonal bi-pyramid, forming a compound hole. The arrangement of these deltahedral units is periodic on GBs, as dictated by the periodicity of adjoining crystals.
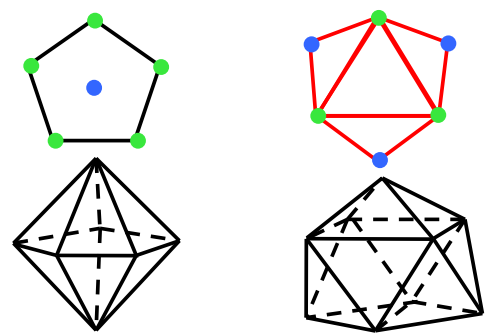

$(5)$
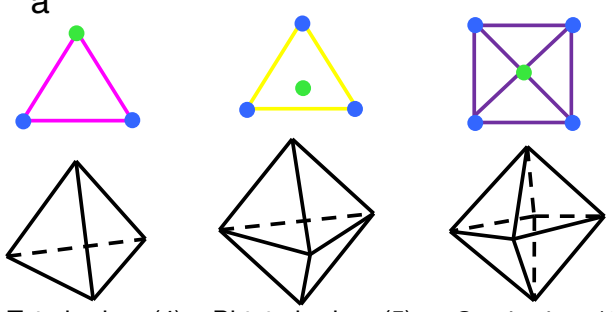

(6) Pentagonal bi-pyramid (7) Capped trigonal prism (8)
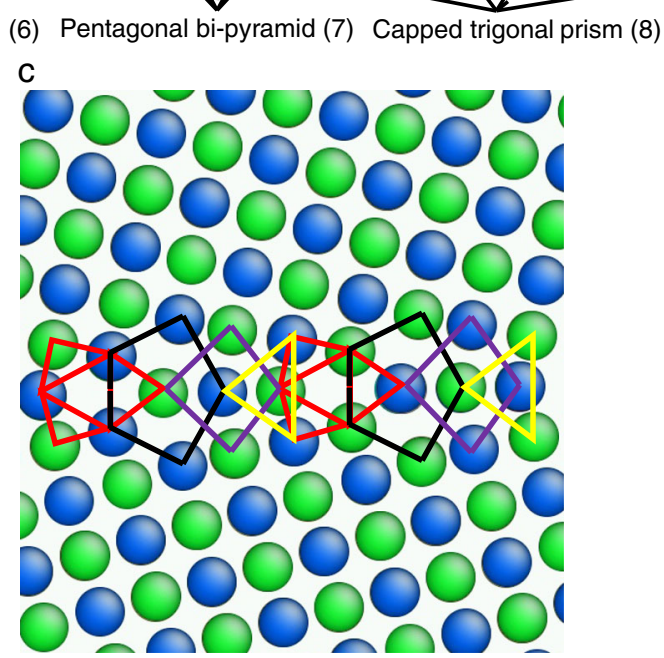

Fig. 1 Symmetric tilt GBs with [001] tilt axis in pure fcc Ni. a Projected and perspective views of deltahedral packing units, with the number of vertices given in the bracket. b A $\sum 5(310)[001] \mathrm{GB}$ is composed of stacks of bi-tetrahedron, pentagonal bi-pyramid, capped trigonal prism; the latter two units form a compound hole. The types of projected deltahedral units in the bulk lattice and at the GB can be recognized from the edge color as defined in a. c A $\sum 17(140)[001] \mathrm{GB}$ is composed of stacks of the above three deltahedral units plus an octahedral unit. Green and blue atoms belong to adjacent (001) planes 
a

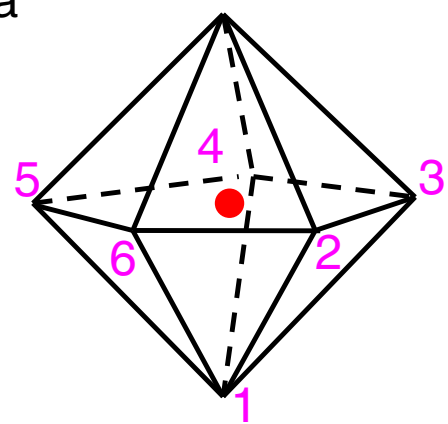

Pentagonal bi-pyramid

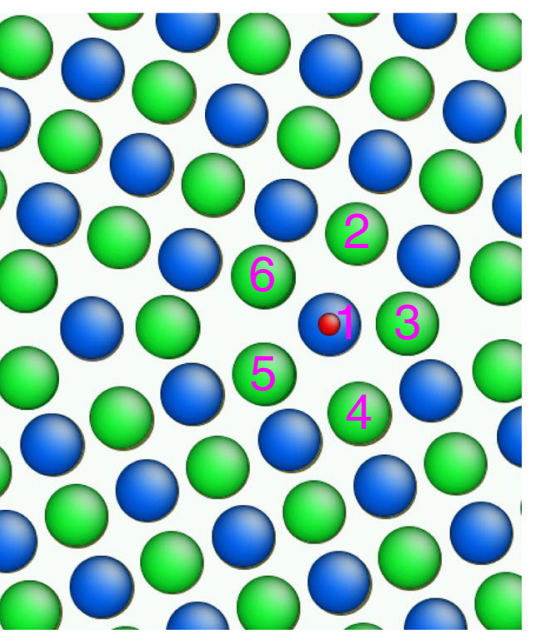

b

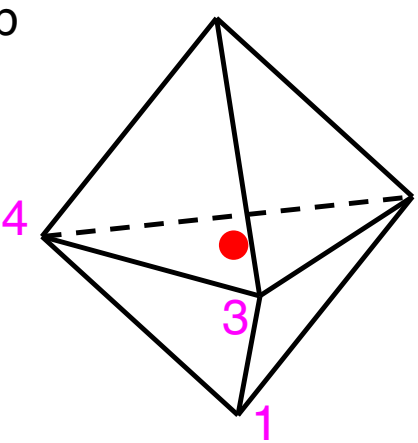

Bi-tetrahedron

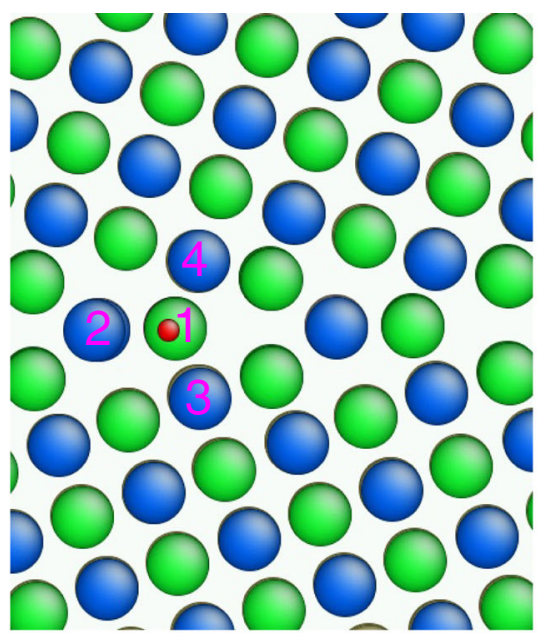

C

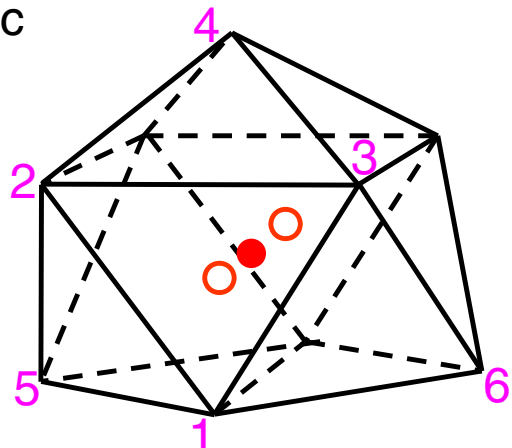

Capped trigonal prism

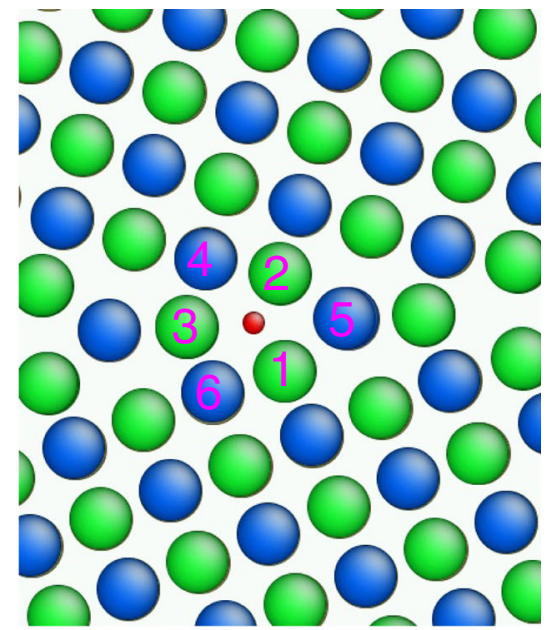

Fig. $2 \mathrm{H}$ adsorption at different interstitial sites of a $\sum 5(310)[001] \mathrm{GB}$. a Schematic of the $\mathrm{H}$ adsorption site (red circle) in a pentagonal bipyramid packing unit (above) and corresponding atomic structure (below). b Same as a except for a bi-tetrahedron packing unit. c Same as a except for a capped trigonal prism packing unit. To facilitate the visualization of deltahedral packing units, the vertices and corresponding atoms of the same kind of deltahedral unit are numbered in the schematics above and the atomic structures below, respectively. Green and blue atoms belongs to adjacent (001) planes

Figure 2 shows the adsorption of a $\mathrm{H}$ atom at the interstitial site of various deltahedral packing units on a $55(310)[001] \mathrm{GB}$; the structure displayed has been relaxed by conjugate gradient energy minimization. In the schematic illustrations (i.e., the upper row of Fig. 2), the solid circle indicates the most energetically favorable binding site for each deltahedral hole. There is only one metastable binding site in the bi-pyramid and bi-tetrahedron holes. In contrast, the capped prism has three metastable binding sites, all located at the central axis of the prism. The two sites indicated by hollow circles deviate from the central cross-sectional plane of the prism by about $20 \%$ of the prism height. Their binding energies are only slightly higher by $0.004 \mathrm{eV}$, such that the three binding sites are approximately equivalent and only one site can be occupied by an interstitial $\mathrm{H}$ atom in this deltahedral unit. The lower row of Fig. 2 shows three different relaxed structures of the $\Sigma 5(310)[001] \mathrm{GB}$, and each structure contains an interstitially adsorbed $\mathrm{H}$ atom in the central hole of the bi-pyramid, bitetrahedron, and capped prism unit, respectively.

Figure 3 shows the atomic structures of a $\mathrm{H}$-segregated $\Sigma 5(310)$ [001] GB before and after cleavage fracture, i.e., separation of adjoining bicrystals at the GB. In Fig. 3a, b, the GB corresponds to an extreme case of full coverage with a monolayer of $\mathrm{H}$ occupying all the interstitial sites. The separated surface is shown in Fig. 3c, where the cleavage boundary plane (as indicated by the red solid line in Fig. 3a) is obtained by energy minimization through direct separation at the GB. It should be noted that the upper and lower separated surfaces in Fig. 3b maintain the mirror symmetry with a relative in-plane shift, and $\mathrm{H}$ atoms are equally partitioned between the two surfaces, such that the surface concentration is reduced to one half of that of the intact, fully covered GB.

\section{$\mathrm{H}$ adsorption and segregation energies}

Table 1 lists the calculated binding energies of $\mathrm{H}$ in vacuum, at various bulk and surface interstitial sites, as compared with available experimental values. Here we denote the molecular binding energy of $\mathrm{H}_{2}$ as $E_{\mathrm{H}}$ per atom. It is computed by the EAM potential as $-2.37 \mathrm{eV}^{33}$ This energy serves as a reference to determine whether the atomic adsorption of $\mathrm{H}$ to various interstitial sites is endothermic or exothermic. The binding energy of $\mathrm{H}$ in the bulk, $\Delta E_{\mathrm{b}}$, is defined as the difference between the energy of the system with and without one $\mathrm{H}$ atom at a trapping site. Table 1 shows that $\mathrm{H}$ binding to an octahedral site $\left(\Delta E_{\mathrm{b}}^{\text {octa }}=-2.20 \mathrm{eV}\right)$ is more energetically favorable than a tetrahedral site $\left(\Delta E_{\mathrm{b}}^{\text {tetra }}=-1.79 \mathrm{eV}\right)$ in the bulk lattice. ${ }^{27}$ These results indicate that $\mathrm{H}_{2}$ dissolution in the bulk is endothermic, since its heat of solution at a favorable octahedral site is positive, i.e., $\Delta E_{\mathrm{b}}^{\text {octa }}-E_{\mathrm{H}}=0.17 \mathrm{eV}$. In contrast, $\mathrm{H}$ adsorption at various sites of the low energy surface is exothermic. For example, the binding energy at the top and three-fold sites of the (111) surface are, respectively, -2.62 and $-2.71 \mathrm{eV}$, both of which are less than $E_{\mathrm{H}}$.

Likewise, we define the binding energy of $\mathrm{H}$ at the $\mathrm{GB}, \Delta E_{\mathrm{g}}^{\mathrm{ad}}$, and at the fracture surface, $\Delta E_{s}^{\text {ad }}$, as the difference between the energy of the system with and without one $\mathrm{H}$ atom at the respective trapping site. Table 2 lists the calculated values of $\Delta E_{\mathrm{g}}^{\mathrm{ad}}$ and $\Delta E_{\mathrm{s}}^{\mathrm{ad}}$. It is seen that for both $\Sigma 5(310)[001]$ and $\Sigma 17(140)[001] \mathrm{GBs}$, the most energetically favorable binding site is the pentagonal bi-pyramidal 

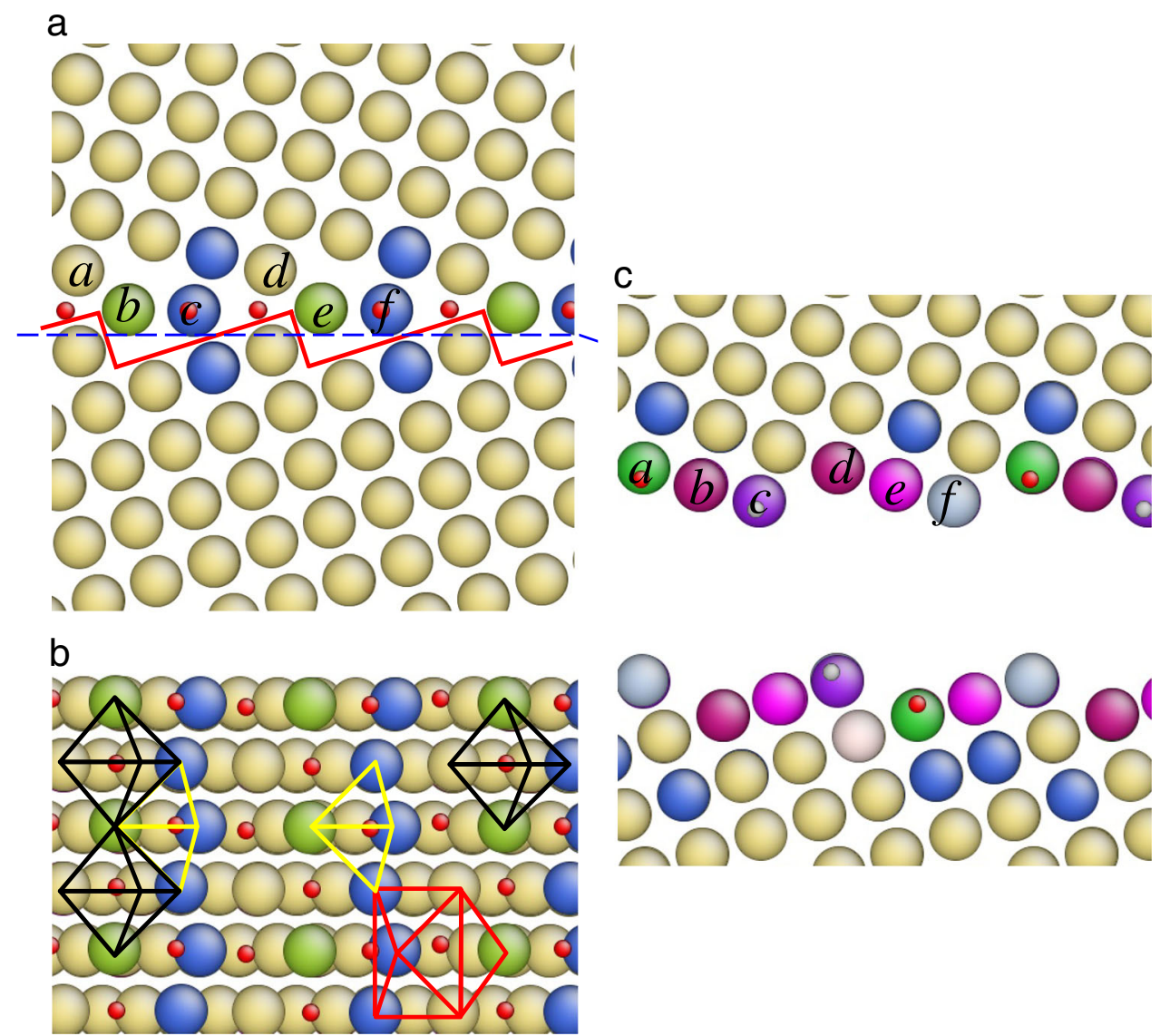

Fig. 3 Atomic structures of the $\sum 5(310)[001] \mathrm{GB}$ with a full H coverage before and after fracture. a The side view of the relaxed GB before fracture, with all the deltahedral holes occupied by interstitial $\mathrm{H}$ atoms, and $\mathbf{b}$ the corresponding cross-sectional view (cut through the blue dashed line in $\mathbf{a}$ ). The colored polyhedra in $\mathbf{b}$ indicate the different packing units at the GB (see definitions in Fig. 1). $\mathbf{c}$ The relaxed structure of the fractured GB; the cleavage plane is indicated by the red solid line in $\mathbf{a}$; surface atoms are marked by $a-f$ for finding the counterparts in $\mathbf{a}$. Atoms are colored by the coordination number [29]

hole. In contrast, the binding energies in the bi-tetrahedral hole are significantly different for two GBs. At the separated surface, the binding energies for the same type of binding sites are very close for the two GBs.

Overall, Table 2 shows that the binding energy of $\mathrm{H}$ at the separated surface $\Delta E_{\mathrm{s}}^{\mathrm{ad}}$ is lower than that at the corresponding $\mathrm{GB}$ site $\Delta E_{\mathrm{g}}^{\mathrm{ad}}$, such that $\Delta E_{\mathrm{g}}^{\mathrm{ad}}-\Delta E_{\mathrm{s}}^{\mathrm{ad}}$ is inherently positive. To understand this trend from the perspective of chemomechanical coupling, we calculate the "chemical" energy change of embedding a $\mathrm{H}$ atom into a rigid atomic network of host $\mathrm{Ni}$ atoms, as well as the "mechanical" energy change due to the elastic relaxation associated with $\mathrm{Ni}-\mathrm{H}$ bonding. As shown in Table 3, the former is much larger than the latter, consistent with the previous quantum mechanical analysis of chemo-mechanical coupling of impurities at GBs. ${ }^{12}$ These results indicate that the positivity of $\Delta E_{\mathrm{a}}^{\mathrm{ad}}-\Delta E_{\mathrm{s}}^{\mathrm{ad}}$ can be mainly attributed to the chemical (electronic) effect of $\mathrm{H}$ binding to Ni. That is, the electron density at the Ni surface is lower than that of the $\mathrm{GB}$, thus enabling the lower embedding energy of $\mathrm{H}$ to the surface. ${ }^{34}$ In contrast, the elastic relaxation due to size mismatch between the small $\mathrm{H}$ atom and the interstice of polyhedral holes plays a minor role.

Since the segregation energies are often used in the thermodynamic theory of interfacial embrittlement, ${ }^{8}$ we convert the $\mathrm{H}$ binding energies in Table 2 to the $\mathrm{H}$ segregation energies from the bulk octahedral site to the interstitial sites at GBs and corresponding fracture surfaces, defined as $\Delta E_{\mathrm{g}}^{\text {seg }}=\Delta E_{\mathrm{g}}^{\mathrm{ad}}-\Delta E_{\mathrm{b}}$ and $\Delta E_{\mathrm{s}}^{\mathrm{seg}}=\Delta E_{\mathrm{s}}^{\mathrm{ad}}-\Delta E_{\mathrm{b}}$, respectively. The calculated segregation energies are also listed in Table 2 . It can be readily verified that
$\Delta E_{\mathrm{g}}^{\mathrm{seg}}-\Delta E_{\mathrm{s}}^{\mathrm{seg}}=\Delta E_{\mathrm{g}}^{\mathrm{ad}}-\Delta E_{\mathrm{s}}^{\mathrm{ad}}$. Note that the results in Tables 1 and 2 are obtained for the dilute limit of low $\mathrm{H}$ concentrations at the GB and separated surface. Our calculations also show that these $\mathrm{H}$ binding energies are nearly identical to the corresponding values in the limit of high $\mathrm{H}$ concentrations, with differences less than $2 \%$. These results indicate that the interaction energies between different $\mathrm{H}$ atoms at the same and different types of deltahedral sites are negligibly small.

\section{Energies of pristine $G B$ and fracture surface}

Table 4 lists the GB energies, $E_{\mathrm{g}}$, and the corresponding surface energies, $E_{\mathrm{s}}$. In addition to the two [001] tilt GBs, we also include two [011] tilt GBs. Also listed is the ideal work of reversibly separating the GBs, $W_{0}=2 E_{\mathrm{s}}-E_{\mathrm{g}}$, as well as that of reversibly separating the lattice planes in the bulk, $W_{0}=2 E_{s}$, giving the surface energy of (111), (100) and (110) planes. We have verified the values of $W_{0}$ in Table 4 by equivalently calculating the area under the stress vs. separation distance curve for GBs and lattice planes studied.

$\mathrm{H}$ embrittlement of GBs-fast fracture

To study the $\mathrm{H}$ embrittlement effects, we first consider the fast separation of GBs at fixed $\mathrm{H}$ concentration, the so-called "fast fracture" limit. According to the Rice-Wang thermodynamic theory of embrittlement of an interface with $n$ types of segregation sites, $^{8}$ the work of separation at constant solute 
Table 2. $\mathrm{H}$ trapping sites and adsorption energies (eV per atom) at $\mathrm{GBs}$ and separated surfaces; $\mathrm{H}$ segregation energies from the bulk lattice to $\mathrm{GBs}$ and separated surfaces, $\Delta E_{\mathrm{g}}^{\mathrm{seg}}=\Delta E_{\mathrm{g}}^{\mathrm{ad}}-\Delta E_{\mathrm{b}}$ and $\Delta E_{\mathrm{s}}^{\mathrm{seg}}=\Delta E_{\mathrm{s}}^{\mathrm{ad}}-\Delta E_{\mathrm{b}}$

\begin{tabular}{|c|c|c|c|c|c|c|c|c|}
\hline \multirow[t]{2}{*}{ Interstitial site } & \multicolumn{4}{|c|}{$\sum 5(310)[001]$} & \multicolumn{4}{|c|}{$\sum 17(140)[001]$} \\
\hline & $\overline{\Delta E_{\mathrm{g}}^{\mathrm{ad}}}$ & $\Delta E_{\mathrm{s}}^{\mathrm{ad}}$ & $\Delta E_{\mathrm{g}}^{\mathrm{seg}}$ & $\Delta E_{\mathrm{s}}^{\mathrm{seg}}$ & $\overline{\Delta E_{g}^{\mathrm{ad}}}$ & $\Delta E_{\mathrm{s}}^{\mathrm{ad}}$ & $\Delta E_{\mathrm{g}}^{\mathrm{seg}}$ & $\Delta E_{\mathrm{s}}^{\mathrm{seg}}$ \\
\hline Pentagonal bi-pyramid & -2.52 & -2.83 & -0.32 & -0.63 & -2.54 & -2.80 & -0.34 & -0.60 \\
\hline Capped trigonal prism & -2.42 & -2.66 & -0.22 & -0.46 & -2.42 & -2.68 & -0.22 & -0.48 \\
\hline Bi-tetrahedron & -2.34 & -2.82 & -0.14 & -0.62 & -2.18 & -2.82 & +0.22 & -0.62 \\
\hline
\end{tabular}

concentration, $W_{\Gamma}$, valid at low temperatures, is

$W_{\Gamma}=W_{0}-\sum_{i} \int_{0}^{\Gamma^{i}}\left[\mu_{\mathrm{g}}^{i}\left(\Gamma^{i}\right)-\mu_{\mathrm{s}}^{i}\left(\frac{\Gamma^{i}}{2}\right)\right] d \Gamma^{i}$

Here, $W_{0}$ is the work of separation of a clean $G B, \Gamma$ is the solute concentration at the $i$-th type interstitial site of the $\mathrm{GB}$, and $\mu_{\mathrm{g}}^{i}$ and $\mu_{\mathrm{s}}^{i}$ are the chemical potentials of solute at the GB and separated surface, respectively. According to Rice and Wang, ${ }^{8}$ for fracture at low temperatures, one can justifiably neglect the entropic contributions to chemical potentials relating to changes in the atomic vibrational spectrum. As a result, the chemical potentials of $\mu_{\mathrm{g}}^{i}$ and $\mu_{\mathrm{s}}^{i}$ in Eq. (2) can be approximated by the corresponding enthalpy $\Delta E_{\mathrm{g}}^{\mathrm{seg}, i}$ and $\Delta E_{\mathrm{s}}^{\mathrm{seg}, i}$, so that

$W_{\Gamma} \approx W_{0}-\sum_{i} \int_{0}^{\Gamma^{i}}\left[\Delta E_{\mathrm{g}}^{\mathrm{seg}, i}\left(\Gamma^{i}\right)-\Delta E_{\mathrm{s}}^{\mathrm{seg}, i}\left(\frac{\Gamma^{i}}{2}\right)\right] d \Gamma^{i}$.

As discussed earlier, the interactions between different $\mathrm{H}$ atoms at the same and different types of deltahedral interstitial sites are negligibly small, such that both $\Delta E_{g}^{\text {seg, } i}$ and $\Delta E_{\mathrm{s}}^{\text {seg, } i}$ can be approximately treated as constant, as assumed in the Langmuir-McLean adsorption isotherm. ${ }^{8}$ It follows that

$W_{\Gamma} \approx W_{0}-\sum_{i}\left(\Delta E_{\mathrm{g}}^{\mathrm{seg}, i}-\Delta E_{\mathrm{s}}^{\mathrm{seg}, i}\right) \Gamma^{i}$

In Eq. (4), the $\mathrm{H}$ concentration at the GB, $\Gamma^{i}$, can be estimated by

$\frac{\Gamma^{i}}{\Gamma_{\mathrm{g}}^{\max , i}-\Gamma^{i}}=x \exp \left(-\frac{\Delta E_{\mathrm{g}}^{\mathrm{seg}, i}}{k T}\right)$

where $\Gamma_{\mathrm{g}}^{\max , i}$ is the maximum concentration of $i$-th type of the polyhedral hole at the GB and $x$ is the fraction of occupied octahedral sites in the bulk. An order-of-magnitude estimate of $\Gamma_{\mathrm{g}}^{\max , i}$ can be made by considering a square network of possible adsorption sites, spaced $0.5 \mathrm{~nm}$ from one another, ${ }^{8}$ giving $\Gamma_{\mathrm{g}}^{\max , i} \approx 4 \times 10^{18} / \mathrm{m}^{2} \approx 7 \times 10^{-6} \mathrm{~mol} / \mathrm{m}^{2}$.

Based on Eqs. (4) and (5), we evaluate the equilibrium $\mathrm{H}$ concentrations and associated embrittlement effects at charging pressures pertinent to the efficient transmission of hydrogen gas, e.g., $20-100 \mathrm{MPa}$ in pipelines. ${ }^{35}$ In a recent experiment by Bechtle et al. ${ }^{5}, \mathrm{H}$ embrittlement was studied with charging pressures between $50-140 \mathrm{MPa}$ and temperatures between $150-200^{\circ} \mathrm{C}$. The corresponding equilibrium $\mathrm{H}$ lattice concentration $x$ was estimated from Sievert's law (see Methods), giving a range of 1000-3000 atomic parts per million (appm) that was consistent with experimental measurements. ${ }^{5}$ Taking a representative lattice concentration in experiment, e.g., $x=3000$ appm when $T=200^{\circ} \mathrm{C}$, we estimate the $\mathrm{H}$ concentrations at GBs using Eqs. (4) and (5) based on the atomistic calculation results of $\mathrm{H}$ segregation energies (Table 2). For the 55(310)[001] GB with $\Gamma_{\mathrm{g}}^{\max , i}=0.85 \times 10^{-5} \mathrm{~mol} / \mathrm{m}^{2}, \Gamma^{i}$ is $0.75 \times 10^{-5} \mathrm{~mol} / \mathrm{m}^{2}$ in the most energetically favorable site of the pentagonal bi-pyramidal hole, giving $88 \%$ coverage of this type of site; in the capped trigonal prism, $\Gamma^{i}=0.34 \times 10^{-5} \mathrm{~mol} / \mathrm{m}^{2}$, giving $40 \%$ coverage; in the bitetrahedron, $\Gamma^{i}=0.07 \times 10^{-5} \mathrm{~mol} / \mathrm{m}^{2}$, giving $8 \%$ coverage. Clearly, these different coverages manifest the segregation equilibrium of $\mathrm{H}$ at multiple polyhedral sites controlled by Eq. (5). ${ }^{11}$

From the above calculated $\mathrm{H}$ concentrations, together with the separation energy of clean boundaries in Table 4, we calculate the work of separation at constant $\mathrm{H}$ concentration, $W_{\Gamma}$, for the $\Sigma 5$ (310)[001] GB based on Eq. (4), and obtain $W_{\Gamma}=3.26 \mathrm{~J} / \mathrm{m}^{2}$ for a Hsegregated $G B$, as opposed to $W_{0}=3.60 \mathrm{~J} / \mathrm{m}^{2}$ for a clean $G B$. Similarly, we evaluate the $\mathrm{H}$ embrittlement effects on different types of high-angle and low-angle GBs with either [001] or [011] tilt axis. The results of $W_{\Gamma}$ are given in Table 5 . Interestingly, the reduction of the various $\mathrm{GB}$ separation energies considered here due to $\mathrm{H}$ segregation, i.e., $\left(W_{\Gamma}-W_{0}\right) / W_{0}$, is around $10 \%$, despite the fact that GBs studied consist of different combinations of polyhedral holes with various spatial arrangements.

$\mathrm{H}$ embrittlement of GBs-slow fracture

The $\mathrm{H}$ embrittlement effect can be drastically increased in the limit of slow separation of a GB at fixed $\mathrm{H}$ chemical potential, the socalled "slow fracture" limit. According to Rice and Wang, ${ }^{8}$ the work of separation at the constant solute chemical potential, $W_{\mu}$, valid at high temperatures, is

$W_{\mu}=W_{0}-\sum_{i} \int_{-\infty}^{\mu}\left[2 \Gamma_{s}^{i}(\mu)-\Gamma_{g}^{i}(\mu)\right] d \mu$,

where the upper integration limit is taken as the solute chemical potential in the bulk $\mu=k T \ln x$. From Eqs. (3) and (6), the work of separation of the GB in the slow and fast separation limits can be related according to

$W_{\ulcorner}-W_{\mu}=\sum_{i} \int_{\Gamma_{0}^{i}}^{2 \Gamma_{\mathrm{s}}^{i}\left(\mu_{0}\right)}\left[\mu_{0}-\mu_{\mathrm{s}}^{i}\left(\frac{\Gamma^{i}}{2}\right)\right] d \Gamma^{i}$

where $\mu_{0}$ is the solute chemical potential in the bulk leading to an equilibrium solute concentration of $\Gamma_{0}^{i}$ at the $G B$, and $\Gamma_{s}^{i}\left(\mu_{0}\right)$ in the integration limit is the solute concentration of the separated surface with the surface chemical potential equal to that in the bulk $\mu_{0}$. To a first approximation and neglect the contributions of vibrational entropy to chemical potentials, the work of separation in the two limits can be estimated by

$W_{\Gamma}-W_{\mu}=\sum_{i}\left(\Delta E_{\mathrm{g}}^{\mathrm{seg}, i}-\Delta E_{\mathrm{s}}^{\mathrm{seg}, i}\right)\left(2 \Gamma_{\mathrm{s}}^{i}-\Gamma_{0}^{i}\right)$,

where the $H$ concentration at the separated surface $\Gamma_{s}^{i}$ is determined by equilibrium of chemical potentials between the bulk and surface, i.e.,

$\frac{\Gamma_{\mathrm{s}}^{i}}{\Gamma_{\mathrm{s}}^{\max , i}-\Gamma_{\mathrm{s}}^{i}}=x \exp \left(-\frac{\Delta E_{\mathrm{s}}^{\mathrm{seg}, i}}{k T}\right)$.

Note that $\Gamma_{s}^{i}$ in Eq. (9) is governed by $\Delta E_{s}^{\text {seg,i } i}$ for separation at constant chemical potential, as opposed to $\Gamma_{s}^{i}$ in Eq. (3) which is controlled by $\Delta E_{b}^{\text {seg,i } i}$ for separation at constant concentration.

Using the same $\mathrm{H}$ lattice concentration $x$ as the case of fast fracture of $\mathrm{GBs}$ at constant $\mathrm{H}$ concentration, we evaluate the $\mathrm{H}$ embrittlement effects on slow fracture at constant chemical 
Table 3. Separation of the $\mathrm{H}$ adsorption energy $E^{\mathrm{ad}}\left(\mathrm{eV}\right.$ per atom) into the chemical contribution $E_{\text {chem }}^{\text {ad }}$ and mechanical contribution $E_{\text {mech }}^{\text {ad }}$, i.e., $E^{\text {ad }}=E_{\text {chem }}^{\text {ad }}+E_{\text {mech }}^{\text {ad }}$. (Pbp: Pentagonal bi-pyramid; Ctp: Capped trigonal prism; Bt: Bi-tetrahedron; Oct: octahedron; Tetr: tetrahedron)

\begin{tabular}{|c|c|c|c|c|c|c|c|c|c|c|c|}
\hline & $\mathrm{Pbp}$ & Ctp & $\mathrm{Bt}$ & $\mathrm{Pbp}$ & Oct & Ctp & $\mathrm{Bt}$ & Oct & Tetr & 3 -fold & Top \\
\hline$E^{\mathrm{ad}}$ & -2.52 & -2.42 & -2.34 & -2.54 & -2.45 & -2.42 & -2.18 & -2.20 & -1.79 & -2.71 & -2.62 \\
\hline$E_{\text {chen }}^{\text {ad }}$ & -2.50 & -2.41 & -2.28 & -2.53 & -2.41 & -2.39 & -2.07 & -2.13 & -1.68 & -1.55 & -1.45 \\
\hline$E_{\text {mech }}^{\text {ad }}$ & -0.02 & -0.01 & -0.06 & -0.01 & -0.04 & -0.03 & -0.11 & -0.07 & -0.11 & -0.16 & -0.17 \\
\hline
\end{tabular}

\begin{tabular}{|c|c|c|c|c|c|c|c|}
\hline & $\begin{array}{l}\sum_{(210)} \\
{[001]}\end{array}$ & $\begin{array}{l}\sum_{(140)} 17 \\
{[001]}\end{array}$ & $\begin{array}{l}\sum_{(111} 11 \\
{[011]}\end{array}$ & $\begin{array}{l}\sum 27 \\
(115) \\
{[011]}\end{array}$ & $\begin{array}{l}\text { (111) } \\
\text { surface }\end{array}$ & $\begin{array}{l}(100) \\
\text { surface }\end{array}$ & $\begin{array}{l}(110) \\
\text { surface }\end{array}$ \\
\hline$E_{\mathrm{g}}$ & 1.12 & 1.15 & 0.28 & 0.62 & - & - & - \\
\hline$E_{\mathrm{s}}$ & 2.36 & 2.31 & 4.57 & 4.55 & 1.92 & 2.06 & 2.34 \\
\hline$w_{0}$ & 3.60 & 3.46 & 4.29 & 3.93 & 3.84 & 4.11 & 4.68 \\
\hline
\end{tabular}

Table 5. Work of separation for (a) pure GBs, $W_{0}$, (b) fast fracture at constant $\mathrm{H}$ concentration, $W_{\Gamma}$, and (c) slow fracture at constant chemical potential, $W_{\mu}$ (unit: $\mathrm{J} / \mathrm{m}^{2}$ )

\begin{tabular}{lllll}
\hline & $\begin{array}{l}\sum 5(310) \\
{[001]}\end{array}$ & $\begin{array}{l}\sum 17(140) \\
{[001]}\end{array}$ & $\begin{array}{l}\sum 11(113) \\
{[011]}\end{array}$ & $\begin{array}{l}\sum 27(115) \\
{[011]}\end{array}$ \\
\hline$W_{0}$ & 3.60 & 3.46 & 4.29 & 3.93 \\
$W_{\Gamma}$ & 3.24 & 3.06 & 3.99 & 3.43 \\
$\left(W_{\Gamma}-W_{0}\right) / W_{0}$ & $-10 \%$ & $-12 \%$ & $-7 \%$ & $-13 \%$ \\
$W_{\mu}$ & 1.94 & 1.64 & 2.27 & 1.44 \\
$\left(W_{\mu}-W_{0}\right) / W_{0}$ & $-46 \%$ & $-53 \%$ & $-47 \%$ & $-63 \%$ \\
\hline
\end{tabular}

potential for high-angle and low-angle GBs with both [001] and [011] tilt axes. The corresponding surface concentration $\Gamma_{\mathrm{s}}^{i}$ at chemical potential equilibrium is calculated from Eq. (9), and then the work of separation $W_{\mu}$ is determined from Eq. (8). The results are also given in Table 5 . It is seen that the reduction of the GB separation energies due to $\mathrm{H}$ segregation, i.e., $\left(W_{\mu}-W_{0}\right) / W_{0}$, is around $50 \%$ in the slow fracture limit, as opposed to about $10 \%$ in the fast fracture limit. Hence, these atomistic calculations demonstrate that the $\mathrm{H}$ embrittlement effects on $\mathrm{GBs}$ is more significant in the slow fracture limit than the fast one.

\section{Electron density effects on $\mathrm{H}$ embrittlement}

To gain a deep understanding of the strength reduction of $\mathrm{H}$ segregated $\mathrm{GBs}$, we analyze the $\mathrm{H}$ adsorption energies at both $\mathrm{GBs}$ and separated surfaces based on the electronic theory of $\mathrm{H}$ alloying in metals. ${ }^{36}$ Figure 4 shows a collection of calculated $\Delta E_{\mathrm{g}}^{\mathrm{ad}, i}$ and $\Delta E_{\mathrm{s}}^{\mathrm{ad}, i}$ at various sites of [001] and [011] GBs studied. These adsorption energies are plotted as a function of local electron density $\rho$ at the corresponding adsorption site. We recall that the electron density $\rho$ is introduced in Eq. (1) and it contributes to the total energy through an embedding effect described by $F(\rho)$. The values of $\rho$ can be evaluated during atomistic calculations with the EAM potential of $\mathrm{Ni}-\mathrm{H}$. One notable feature in Fig. 4 is that there exists a finite energy gap (no less than $0.14 \mathrm{eV}$ ) between $\Delta E_{\mathrm{g}}^{\mathrm{ad}, i}$ and $\Delta E_{\mathrm{s}}^{\mathrm{ad}, i}$, irrespective of the type of adsorption sites. Also note that the adsorption energies at various GB trapping sites $\Delta E_{\mathrm{g}}^{\mathrm{ad}, i}$ (hollow symbols) exhibit a relatively large variation, while those at different surface trapping sites $\Delta E_{\mathrm{s}}^{\text {ad, } i}$

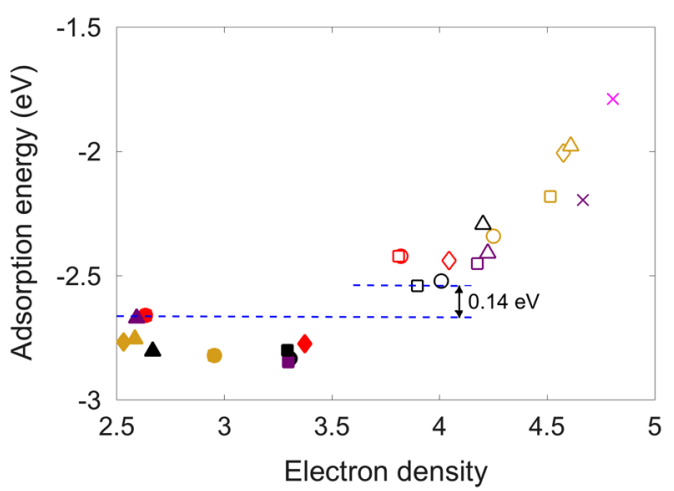

Fig. $4 \mathrm{H}$ adsorption energy vs. local electron density at different adsorption sites. The adsorption sites in the bulk lattice are represented by cross symbols. The adsorption sites on the GBs and corresponding separated surfaces are respectively represented by hollow and solid symbols, including $\sum 5(310)[001]$ (circle), $\sum 17(140)$ [001](square), $\sum 11$ (113)[011](diamond), and $\sum 27(115)[011]$ (triangle). The color of symbols indicates the type of deltahedral units, with the same edge coloring scheme in Fig. 1a, i.e., pink: tetrahedron; yellow: bi-tetrahedron; purple: for octahedron; black: pentagonal bi-pyramid; and red: capped trigonal prism

(solid symbols) are much closer to each other. These features can be rationalized in terms of the $\mathrm{H}$ alloying behavior in metals. ${ }^{30}$ Specifically, the interaction energy between $\mathrm{H}$ and the host transition metal such as $\mathrm{Ni}$ is mainly governed by the embedding energy $F(\rho)$, which is a function of local electron density $\rho$ at the adsorption site determined by the host metal. ${ }^{30}$ The nearly constant value of $\Delta E_{\mathrm{s}}^{\text {ad, } i}$ arises mainly because of gradual attenuation of the electron density in the free space outside the surface, enabling a $\mathrm{H}$ atom to find an optimal (minimal) embedding energy. In contrast, the range of variation of the electron density at an internal interstitial site in a GB is dictated by the geometrical constraint of surrounding atoms that make up the polyhedral packing unit. As a result, the corresponding embedding energy cannot be optimized to the minimum. This gives rise to a relatively large range of $\Delta E_{\mathrm{a}, i}^{\mathrm{a}}$ due to the variation of local atomic geometries of polyhedral holes. As such, a finite energy gap exists between $\Delta E_{\mathrm{g}}^{\mathrm{ad}, i}$ and $\Delta E_{\mathrm{s}}^{\mathrm{ad}, i}$.

Based on the foregoing analysis of adsorption energies and effects of local electron densities, we can further rationalize the extent of reduction of the GB strength by $\mathrm{H}$ segregation. Taking the fast fracture at fixed $\mathrm{H}$ concentration as an example, we recall from Eq. (4) that $\mathrm{H}$ embrittlement is controlled by the collective effect of multiple segregation sites; and for each type of site, the extent of embrittlement is determined by product between the segregation energy difference, $\Delta E_{g}^{\text {seg, } i}-\Delta E_{s}^{\text {seg, } i}$, and the $\mathrm{H}$ concentration at the GB, $\Gamma^{i}$. The former is equal to the adsorption energy difference, $\Delta E_{\mathrm{g}}^{\mathrm{ad}, i}-\Delta E_{\mathrm{s}}^{\mathrm{ad}, i}$. According to Eq. (5) the latter is governed by $\Delta E_{\mathrm{g}}^{\text {seg, } i}$ and equivalently by $\Delta E_{\mathrm{g}}^{\mathrm{ad}, i} i$ in the exponential, under a given charging load and accordingly a lattice concentration $x$. Since a small variation of $\Delta E_{q}^{\text {ad, } i}$ can be markedly magnified by the exponential in Eq. (5), $\Delta E_{\mathrm{g}}^{\text {ad, }}$, will affect the embrittlement 
effect primarily through $\Gamma^{i}$. For the $\mathrm{Ni}-\mathrm{H}$ system, $\Delta E_{\mathrm{g}}^{\mathrm{ad}, i}$ is negative. As a result, at different $\mathrm{H}$ adsorption sites, if the magnitude of $\Delta E_{\mathrm{g}}^{\mathrm{ad}, i}$ is large, $\Gamma^{i}$ will be high, and the induced decrease of work of separation would be significant. However, the variation of $\Delta E_{\mathrm{g}}^{\mathrm{ad}, i}$ is limited. This is because of the constraints of atomic structure on the allowable electron densities within polyhedral holes at GBs as discussed earlier. It follows that the most embrittling site is the one with a maximum magnitude of $\Delta E_{\mathrm{a}}^{\mathrm{ad}, i}$, with the corresponding $\Delta E_{\mathrm{g}}^{\mathrm{seg}, i}-\Delta E_{\mathrm{s}}^{\mathrm{seg}, i} \approx 0.25 \mathrm{eV}$ per $\mathrm{H}$ atom. We also note that the limit of $\Gamma^{i}$ is set by the limit of $\Gamma_{\mathrm{g}}^{\mathrm{max}, i}$. That is, under the high charging load studied in this work, $\Gamma^{i}$ at the site with the maximum magnitude of $\Delta E_{\mathrm{g}}^{\mathrm{ad}, i}$ approaches the saturation limit of $\Gamma_{\mathrm{g}}^{\max , i} \approx 4 \times 10^{18} \mathrm{~m}^{-2}$, which is intrinsically determined by the atomic structure of GBs or simply by the lattice constant, as discussed earlier. From the above estimation, we conclude that the maximum product between $\Delta E_{\mathrm{g}, i}^{\mathrm{ad}, i}-\Delta E_{\mathrm{s}}^{\mathrm{ad}, i}$ and $\Gamma^{i}\left(\approx \Gamma_{\mathrm{g}}^{\mathrm{max}, i}\right)$ in Eq. (4) is approximately $0.16 \mathrm{~J} / \mathrm{m}^{2}$, which corresponds to a reduction of the work of separation of pure GBs by about $5 \%$ (taking a representative value of $W_{0}=3 \mathrm{~J} / \mathrm{m}^{2}$ ). This value gives a reasonable order-of-magnitude estimate to the reduction of $W_{0}$ at $\mathrm{H}$-segregated GBs. In addition, because of the high charging load considered, the weakening effects due to $\mathrm{H}$ adsorption at other types of interstitial sites can make comparable contributions, as shown by detailed numerical examples given earlier, so that the total weakening amounts to about $10 \%$ reduction of $W_{0}$ in the fast fracture limit.

Finally, we note that the above analysis of the extent of $\mathrm{H}$ embrittlement is given for the case of fast fracture. Likewise, the $\mathrm{H}$ adsorption energies at GBs and separated surfaces, as governed by local electron densities and atomic structures, should also dictate the embrittlement extent for the case of slow fracture. A similar analysis can be given along the same line, but is more involved under constant $\mathrm{H}$ chemical potential and thus worth studying in the future.

\section{DISCUSSION}

We have performed an atomistic study of $\mathrm{H}$ embrittlement of GBs in Ni by combining the geometrical analysis of polyhedral packing units for $\mathrm{H}$ interstitial adsorption, atomistic calculation of $\mathrm{H}$ segregation energies, and thermodynamic theory of interfacial embrittlement. The $\mathrm{H}$ embrittlement effects are quantitatively evaluated for symmetric tilt GBs of $\Sigma 5(310)[001], \Sigma 17(140)[001]$, $\Sigma 11(113)[011]$ and $\Sigma 27(115)[011]$. Our atomistic results show that the strength reduction of $\mathrm{H}$-segregated $\mathrm{GBs}$ in $\mathrm{Ni}$ is around $10 \%$ in the limit of fast GB fracture at constant $\mathrm{H}$ concentration, while the reduction of the GB strength is around $50 \%$ in the limit of slow GB fracture at constant $\mathrm{H}$ chemical potential. The extent of reduction of the $\mathrm{GB}$ strength due to $\mathrm{H}$ segregation is rationalized in terms of local electron densities and their effects on $\mathrm{H}$ adsorption energies at GBs and separated surfaces.

Broadly, our work is built upon a powerful geometrical approach for finding all the possible polyhedral interstitial sites of impurity adsorption, and thus provides an atomistically-based thermodynamic framework to quantify the interfacial embrittlement by impurity segregation in metals. While only symmetric tilt GBs are studied in this paper, the geometrical analysis in terms of polyhedral packing units can be applied to general GBs in metals, including tilt and twist, symmetric and asymmetric boundaries. Note that only the monolayer $\mathrm{H}$ coverage at the GB layer is considered here. Depending on the GB structure, various interstitial sites close to the GB layer (e.g., one or two layers away from the GB layer) can be energetically favored for $\mathrm{H}$ absorption as well. Their effects on GB embrittlement warrant a systematic study in the future. It should be emphasized that the present study is focused on direct $\mathrm{H}$ embrittlement effects on GBs in the absence of defects such as vacancies (i.e., large free volumes) and dislocations at GBs. While the reduction of GB strengths at high
$\mathrm{H}$ converges falls in the range of only a few tens of percent, such weakening of GBs could still suffice to cause a transition of overall fracture from the ductile mode of dimpled fracture without $\mathrm{H}$ charging to the brittle mode of intergranular fracture with $\mathrm{H}$ charging, as observed in experiments. ${ }^{5}$ One possible mechanism underlying such transition could be plasticity-mediated atomic decohesion, involving the so-called "valve" effect of coupling a small variation of cohesive strength with a large change of plastic dissipation during fracture. $8,37,38$ To confirm the efficacy of this valve effect, it is necessary to extend the present atomisticallybased thermodynamic model to address the kinetic aspects of $\mathrm{H}$ diffusion and crack growth at GBs, as well as the coupling effects of $\mathrm{H}$-enhanced localized plasticity (HELP), $\mathrm{H}$-enhanced decohesion (HEDE), H-enhanced vacancy stabilization (HEVS) ${ }^{39,} 40$ for realistic geometries of three-dimensional cracks and polycrystals on the time scale relevant to laboratory experiments. ${ }^{41-43}$

\section{METHODS}

\section{Evaluation of $\mathrm{H}$ lattice concentration}

We describe how the $\mathrm{H}$ lattice concentrations can be estimated from the hydrogen charging pressure, according to Bechtle et al. ${ }^{5}$ One can relate the hydrogen gas pressure to the lattice concentration $x$ based on Sievert's law, $x=S f^{1 / 2}$. Here the Sievert's parameter $S$ is given by $S=S_{0} \exp \left(-E_{0} / k T\right)$, where $E_{0}$ is the heat of solution, i.e., the adsorption energy in the bulk ( $E_{0}=0.17 \mathrm{eV}$ from atomistic calculations vs. $E_{0}=0.16 \mathrm{eV}$ from experiment); $S_{0}$ is the solubility constant that can be measured from experiment $\left(S_{0}=9.88 \times 10^{3}\right.$ appm $\mathrm{MPa}^{-1 / 2}$ for $\left.\mathrm{H} \mathrm{in} \mathrm{Ni)}\right) ;$ is the fugacity of the hydrogen gas, given by $f=P \exp (P b / k T)$, where $b$ is a constant in the Abel-Noble equation of state accounting for the non-ideal gas behavior of a finite sized molecule.

Data availability

The data that support the findings of this study are available from the corresponding author upon reasonable request.

\section{ACKNOWLEDGEMENTS}

T.Z. and D.L.M acknowledge partial support from QuesTek LLC to study hydrogen effects in metals. T.Z. acknowledges support by NSF Grant CMMI 0758554. D.L.M. acknowledges support of the Carter N. Paden, Jr. Distinguished Chair in Metals Processing. We thank Dr. Mike Baskes for his help in implementing the interatomic potential of $\mathrm{Ni}-\mathrm{H}$.

\section{AUTHOR CONTRIBUTIONS}

T.Z. and D.L.M. designed the research. S.H. and D.C. performed the research. All the authors wrote the paper.

\section{ADDITIONAL INFORMATION}

Competing interests: The authors declare that they have no competing financial interests.

Publisher's note: Springer Nature remains neutral with regard to jurisdictional claims in published maps and institutional affiliations.

\section{REFERENCES}

1. Service, R. F. The hydrogen backlash. Science 305, 958-961 (2004).

2. Myers, S. M. et al. Hydrogen interactions with defects in crystalline solids. Rev. Mod. Phys. 64, 559-617 (1992).

3. Birnbaum, H. K., Robertson, I. M., Sofronis, P. \& Teter, D. in Corrosion Deformation Interactions CDI'96 (ed Magnin, T.) 172-195 (The Institute of Materials, 1997).

4. Murakami, Y. The effect of hydrogen on fatigue properties of metals used for fuel cell system. Int. J. Fract. 138, 167-195 (2006).

5. Bechtle, S., Kumar, M., Somerday, B. P., Launey, M. E. \& Ritchie, R. O. Grainboundary engineering markedly reduces susceptibility to intergranular hydrogen embrittlement in metallic materials. Acta Mater. 57, 4148-4157 (2009).

6. Rice, J. R. in Effect of Hydrogen on Behavior of Materials (eds Thompson, A. W. \& Bernstein, I. M.) 455-466 (The Metallurgical Society of AIME, 1976). 
7. Hirth, J. P. \& Rice, J. R. On the thermodynamics of adsorption at interfaces as it influences decohesion. Metall. Trans. A 11, 1501-1511 (1980).

8. Rice, J. R. \& Wang, J. S. Embrittlement of interfaces by solute segregation. Mater. Sci. Eng. A 107, 23-40 (1989).

9. Mishin, Y., Sofronis, P. \& Bassani, J. L. Thermodynamic and kinetic aspects of interfacial decohesion. Acta Mater. 50, 3609-3622 (2002).

10. Baskes, M. I. \& Vitek, V. Trapping of hydrogen and hellium at grain-boundaries in nickel - An atomistic study. Metall. Trans. A 16, 1625-1631 (1985).

11. Mutschele, T. \& Kirchheim, R. Segregation and diffusion of hydrogen in grainboundaries of palladium. Scr. Metall. 21, 135-140 (1987).

12. Geng, W. T., Freeman, A. J., Olson, G. B., Tateyama, Y. \& Ohno, T. Hydrogenpromoted grain boundary embrittlement and vacancy activity in metals: insights from ab initio total energy calculatons. Mater. Trans. 46, 756-760 (2005).

13. Van der Ven, A. \& Ceder, G. The thermodynamics of decohesion. Acta Mater. 52, 1223-1235 (2004)

14. Jiang, D. E. \& Carter, E. A. First principles assessment of ideal fracture energies of materials with mobile impurities: implications for hydrogen embrittlement of metals. Acta Mater. 52, 4801-4807 (2004).

15. Lu, G. \& Kaxiras, E. Hydrogen embrittlement of aluminum: the crucial role of vacancies. Phys. Rev. Lett. 94, 155501 (2005).

16. Zhu, T. \& Li, J. Ultra-strength materials. Prog. Mater. Sci. 55, 710-757 (2010).

17. Song, J. \& Curtin, W. A. A nanoscale mechanism of hydrogen embrittlement in metals. Acta Mater. 59, 1557-1569 (2011).

18. Song, J. \& Curtin, W. A. Atomic mechanism and prediction of hydrogen embrittlement in iron. Nat. Mater. 12, 145-151 (2013).

19. Song, J. \& Curtin, W. A. Mechanisms of hydrogen-enhanced localized plasticity: an atomistic study using alpha-Fe as a model system. Acta Mater. 68, 61-69 (2014).

20. Kirchheim, R., Somerday, B. \& Sofronis, P. Chemomechanical effects on the separation of interfaces occurring during fracture with emphasis on the hydrogen-iron and hydrogen-nickel system. Acta Mater. 99, 87-98 (2015).

21. Wang, S., Martin, M. L., Robertson, I. M. \& Sofronis, P. Effect of hydrogen environment on the separation of Fe grain boundaries. Acta Mater. 107, 279-288 (2016).

22. Tehranchi, A. \& Curtin, W. A. Atomistic study of hydrogen embrittlement of grain boundaries in nickel: I. Fracture. J. Mech. Phys. Solids 101, 150-165 (2017).

23. Sutton, A. P. \& Balluffi, R. W. Interfaces in Crystalline Materials (Oxford University Press, 1995)

24. Ashby, M. F., Spaepen, F. \& Williams, S. Structure of grain-boundaries described as a packing of polyhedra. Acta Metall. 26, 1647-1663 (1978).

25. Pond, R. C., Vitek, V. \& Smith, D. A. Acta Crystallogr. Sect. A. Acta Crystallogr. A 35 , 689-693 (1979).

26. McDowell, D. L. Viscoplasticity of heterogeneous metallic materials. Mater. Sci. Eng. R Rep. 62, 67-123 (2008).

27. Angelo, J. E., Moody, N. R. \& Baskes, M. I. Trapping of hydrogen to lattice-defects in nickel. Model. Simul. Mat. Sci. Eng. 3, 289-307 (1995).

28. Baskes, M. I., Sha, X. W., Angelo, J. E. \& Moody, N. R. Trapping of hydrogen to lattice defects in nickel. Model. Simul. Mat. Sci. Eng. 5, 651-652 (1997).

29. National Institute of Standards and Technology (NIST). Interatomic Potentials Repository Project http://www.ctcms.nist.gov/potentials/Ni-Al-H.html (2017).
30. Cottrell, A. H. Concepts of the Electron Theory of Alloys. (IOM Communications Ltd, 1998).

31. Zhou, X., Marchand, D., McDowell, D. L., Zhu, T. \& Song, J. Chemomechanical origin of hydrogen trapping at grain boundaries in fcc metals. Phys. Rev. Lett. 116, 075502 (2016).

32. Li, J. AtomEye: an efficient atomistic configuration viewer. Model. Simul. Mat. Sci. Eng. 11, 173-177 (2003).

33. Foiles, S. M., Baskes, M. I., Melius, C. F. \& Daw, M. S. Calculation of hydrogen dissociation pathways on nickel using the embedded atom method. J. Less Common Met. 130, 465-473 (1987).

34. Cottrell, A. H. Strength of grain-boundaries in impure metals. Mater. Sci. Technol. 6, 325-329 (1990).

35. Novak, P., Yuan, R., Somerday, B. P., Sofronis, P. \& Ritchie, R. O. A statistical, physical-based, micro-mechanical model of hydrogen-induced intergranular fracture in steel. J. Mech. Phys. Solids. 58, 206-226 (2010).

36. Cottrell, A. H. Strengthening of grain boundaries by segregated interstitials in iron. Mater. Sci. Technol. 6, 121-123 (1990).

37. Jokl, M. L., Vitek, V. \& McMahon, C. J. A microscopic theory of brittle-fracture in deformable solids - a relation between ideal work to fracture and plastic work. Acta Metall. 28, 1479-1488 (1980).

38. Suo, Z., Shih, C. F. \& Varias, A. G. A theory for cleavage cracking in the presence of plastic-flow. Acta Metall. Mater. 41, 1551-1557 (1993).

39. Robertson, I. M. et al. Hydrogen embrittlement understood. Metall. Mater. Trans. $B$ 46, 1085-1103 (2015).

40. Neeraj, T., Srinivasan, R. \& Li, J. Hydrogen embrittlement of ferritic steels: observations on deformation microstructure, nanoscale dimples and failure by nanovoiding. Acta Mater. 60, 5160-5171 (2012).

41. Zhu, T., Li, J. \& Yip, S. Atomistic study of dislocation loop emission from a crack tip. Phys. Rev. Lett. 93, 025503 (2004).

42. Zhu, T., Li, J. \& Yip, S. Atomistic configurations and energetics of crack extension in silicon. Phys. Rev. Lett. 93, 205504 (2004).

43. Warner, D. H., Curtin, W. A. \& Qu, S. Rate dependence of crack-tip processes predicts twinning trends in f.c.c. metals. Nat. Mater. 6, 876-881 (2007).

\section{(1)}

Open Access This article is licensed under a Creative Commons Attribution 4.0 International License, which permits use, sharing, adaptation, distribution and reproduction in any medium or format, as long as you give appropriate credit to the original author(s) and the source, provide a link to the Creative Commons license, and indicate if changes were made. The images or other third party material in this article are included in the article's Creative Commons license, unless indicated otherwise in a credit line to the material. If material is not included in the article's Creative Commons license and your intended use is not permitted by statutory regulation or exceeds the permitted use, you will need to obtain permission directly from the copyright holder. To view a copy of this license, visit http://creativecommons. org/licenses/by/4.0/.

(c) The Author(s) 2017 